This item was submitted to Loughborough's Research Repository by the author.

Items in Figshare are protected by copyright, with all rights reserved, unless otherwise indicated.

\title{
Output selection under control and fault detectability considerations
}

PLEASE CITE THE PUBLISHED VERSION

PUBLISHER

(C) Institute of Electrical and Electronics Engineers (IEEE)

LICENCE

CC BY-NC-ND 4.0

\section{REPOSITORY RECORD}

Li, Zhenhai, Argyrios C. Zolotas, I.M. Jaimoukha, K.M. Grigoriadis, Konstantinos Michail, and John T. Pearson. 2019. "Output Selection Under Control and Fault Detectability Considerations". figshare.

https://hdl.handle.net/2134/3579. 
This item was submitted to Loughborough's Institutional Repository by the author and is made available under the following Creative Commons Licence conditions.

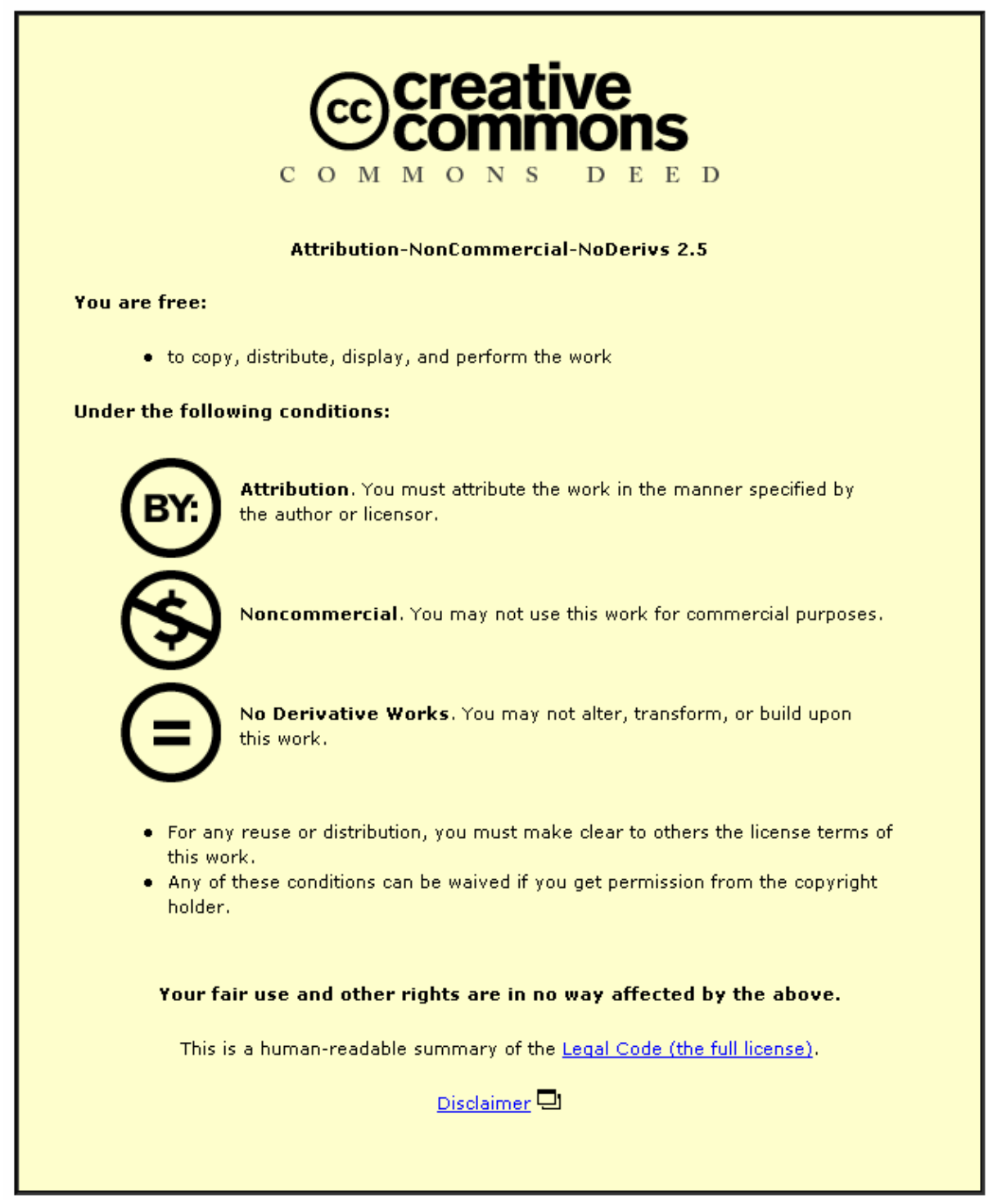

For the full text of this licence, please go to: http://creativecommons.org/licenses/by-nc-nd/2.5/ 


\title{
Output Selection Under Control and Fault Detectability Considerations
}

\author{
Z. Li ${ }^{*}$ A. C. Zolotas*, I. M. Jaimoukha ${ }^{\dagger}$, K. M. Grigoriadis ${ }^{\ddagger}$, K. Michail ${ }^{*}$, J. T. Pearson ${ }^{\S}$ \\ ${ }^{*}$ Department of Electronic and Electrical Engineering, Loughborough University, UK \\ ${ }^{\dagger}$ Department of Electrical and Electronic Engineering, Imperial College London, UK \\ ${ }^{\ddagger}$ Department of Mechanical Engineering, University of Houston, USA \\ $\S$ Systems Engineering Innovation Centre (SEIC), BAE Systems, UK
}

\begin{abstract}
In a variety of practical engineering systems, i.e. aerospace, mechanical systems, railway vehicle systems, for a given requirement the range of possible locations for sensors is usually known, with the practical engineering issue of optimizing their location. Input-Output selection/placement for control systems has been widely researched in particular under fault-free conditions. In this paper we discuss on the feasibility of an (output) sensor selection scheme in a closed-loop framework based on both control performance and fault detectability metrics. The selection of sensors is based upon both closed-loop control and fault detection objectives by solving a mixed $\mathcal{H}_{-} / \mathcal{H}_{\infty}$ optimization problem for each group of sensors available via Linear Matrix Inequalities (LMI). The efficacy of the scheme is illustrated via a numerical example.
\end{abstract}

\section{INTRODUCTION}

Modern control and monitoring systems that involve a large number of actuators and sensors that are prone to failure are becoming rather complex and demanding in terms of maintenance. In a variety of practical engineering systems (aerospace, electro-mechanical systems, railway vehicle systems) for a given requirement the range of possible locations for sensors is usually known, the practical engineering issue is either to minimize the number of sensors to achieve a particular level of fault tolerance, or to optimise the location of such a number of sensors. The role of sensors in the overall process is undoubtedly very important. The signals provided by the sensory equipment comprise the data that is utilised by the control, monitoring and diagnostic algorithms. The choice of the number, location and type of sensors (and undoubtedly that of the actuators) has a significant impact on the performance, the complexity and the overall cost of the system. In this paper the primary focus is that of optimised sensor selection for efficient robustness properties of the system, assuming a consistent controller design, with relation to fault detectability issues.

The general problem of sensor/actuator placement (pairs) in the area of feedback control has received significant attention during the past two decades. The sensor/actuator location is optimised in order to increase, from a control perspective, the relative controllability and

\footnotetext{
${ }^{1}$ This research was supported in part under the EPSRC project Grant Ref. EP/D063965/1 and BAE Systems, and also has been partially supported by the Ministry of Defence (MoD) through the Data \& Information Fusion Defence Technology Centre.
}

observability of the important system modes. Moreover, an further survey on input/output selection methods can be found in [1] (and references within), where a review and some assessment issues are presented according to the desired properties relating to each selection method. However, the core of this and of other previous works is on fault-free environments.

In the area of fault detection and isolation there is some work on sensor placement with an early paper [2] discussing on optimal sensor location for monitoring eigen-structures of multivariable systems, as well as in other papers [3], [4] although still concentrating on probabilistic and statistical approaches. In addition, work on the integration of control and fault detection and fault tolerant feedback control can be found in [5], [6], although this concentrates on issues related to the solution of the control and fault tolerant subproblems rather than particular sensor selection.

In this paper we investigate a framework which incorporates sensor faults and determine an appropriate set of criteria for the optimal selection relating to fault detectability. An iterative approach is employed to lock on the sensor combination which provides the best mixed $\mathcal{H}_{\infty}$ performance for control and $\mathcal{H}_{-} / \mathcal{H}_{\infty}$ performance for fault detection. Each step in the iteration is solved by Linear Matrix Inequalities (LMIs). The $\mathcal{H}_{\infty}$ performance is employed to cover a worst case control purpose and the $\mathcal{H}_{-} / \mathcal{H}_{\infty}$ performance guarantees fault indication to be maximally insensitive to disturbances for a given minimum level of sensitivity to faults.

The paper is organised as follows: Section II gives a performance formulation of the output selection problem with consideration of fault detectability. Section III transforms the formulation into a state space framework under certain assumptions via LMI solutions and compares the performance index under different output combinations. Finally, a numerical example is given in Section IV while concluding remarks are made and future research directions are mapped out in Section V.

The notation we use is mostly standard and is summarized next for convenience.The set of real (complex) $n \times m$ matrices is denoted by $\mathcal{R}^{n \times m}\left(\mathcal{C}^{n \times m}\right)$. For $A \in \mathcal{C}^{n \times m}$ we use the notation $A^{T}$ and $A^{\prime}$ to denote the transpose and complex conjugate transpose, respectively. For $A=$ $A^{\prime} \in \mathcal{C}^{n \times n}, A \geq 0$ denotes that $A$ is positive semidefinite 


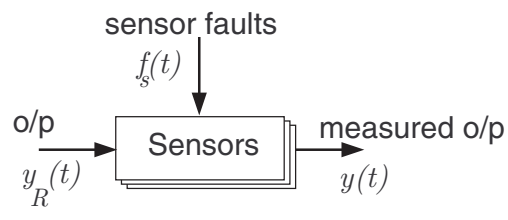

Fig. 2. Additive sensor faults identity matrix is denoted as $I_{n}$ and the $n \times m$ null matrix is denoted as $0_{n, m}$ with the subscripts occasionally dropped if they can be inferred from context.

$\mathcal{R}(s)^{m \times p}$ denotes the set of all $m \times p$ real rational matrix functions of $s . \mathcal{L}_{\infty}^{m \times p}$ denotes the space of $m \times p$ matrix functions with entries bounded on the extended imaginary axis $j \mathcal{R}_{e}$. The subspace $\mathcal{H}_{\infty}^{m \times p} \subset \mathcal{L}_{\infty}^{m \times p}$ denotes matrix functions analytic in the closed righthalf of the complex plane. A prefix $\mathcal{R}$ denotes a real rational function, so that $\mathcal{R} \mathcal{H}_{\infty}^{m \times p}$ denotes the set of all $m \times p$ stable real rational matrix functions of $s$. For $G(s) \in \mathcal{R} \mathcal{H}_{\infty}^{m \times p}$ we define

$$
\|G\|_{\infty}=\sup _{\omega \in \mathcal{R}} \bar{\sigma}(G(j \omega)), \quad\|G\|_{-}=\inf _{\omega \in \mathcal{R}} \underline{\sigma}(G(j \omega)) .
$$

For $G(s) \in \mathcal{R} \mathcal{L}_{\infty}^{m \times p}$, we define $G^{\sim}(s)=G(-s)^{T}$ to be the para-Hermitian complex conjugate transpose of $G(s)$. A square matrix function $G(s) \in \mathcal{R} \mathcal{H}_{\infty}^{m \times m}$ is called inner if $G^{\sim}(s) G(s)=I_{m}$.

\section{Output Selection Problem Formulation}

The proposed output selection scheme based upon a $\mathcal{H}_{\infty}$ control performance index of closed-loop system transfer functions, integrated with a fault detection filter as depicted in Fig. 1.



Fig. 1. Generalized regulator with fault detection filter

Note that $w(s)$ characterises any exogenous inputs entering the system, $u(s)$ is the fixed set of control inputs, $y(s)$ the measurements (their number varies depending on the scenario considered) and $z(s)$ is the vector of regulated outputs (these can be related to $\infty$-norm or 2 norm or both types of the aforementioned norms). For the purposes of this work we consider only $\infty$-norm regulation, i.e. $z_{\infty}(s)$, for the control objectives.

Next (and an important step towards fault tolerance) is to incorporate sensor fault detectability. The aim here is to cover additive sensor faults, Fig. 2, which affect the output directly as the dotted line in Fig. 1 indicates, i.e. $y(t)=y_{R}(t)+f_{s}(t)$
Note that sensor faults can also have direct channels to state dynamics or can be transferred into pseudo-actuator faults when necessary [7], [8]. Hence, a generalized faulty system is given by

$$
\begin{aligned}
& \dot{x}(t)=A x(t)+B_{1} w(t)+B_{2} u(t)+B_{f} f(t) \\
& y(t)=C_{y} x(t)+D_{y 1} w(t)+D_{f} f(t)
\end{aligned}
$$

where $B_{f}$ and $D_{f}$ are well-defined distribution matrices with appropriate dimensions.

Our objective is then to explore the channels by which a certain level of system performance is maintained and faults have most effect on the residual signal such that potential faults can be appropriately indicated. The selection of sensors can then be improved for post-fault configuration and fault tolerance.

First consider a faulty Linear Time Invariant (LTI) dynamic system subject to both disturbances and faults as follows

$$
\begin{aligned}
\dot{x}(t) & =A x(t)+B_{1} w(t)+B_{2} u(t)+B_{f} f(t) \\
z_{\infty}(t) & =C_{\infty} x(t)+D_{\infty 1} w(t)+D_{\infty 2} u(t) \\
y(t) & =C_{y} x(t)+D_{y 1} w(t)+D_{f} f(t)
\end{aligned}
$$

where $x(t) \in \mathcal{R}^{n_{p}}, u(t) \in \mathcal{R}^{n_{p u}}$ and $y(t) \in \mathcal{R}^{n_{p y}}$ are the state, input and output vectors, respectively and $w(t) \in \mathcal{R}^{n_{w}}$ is the disturbance vector. The energy of the output signal $z_{\infty}(t) \in \mathcal{R}^{n_{p 1}}$ is bounded for finite energy input signals by regulating the $\mathcal{H}_{\infty}$ norm of the system input-output gain (robustness metric). Here, $B_{1} \in \mathcal{R}^{n_{p} \times n_{w}}, D_{\infty 1} \in \mathcal{R}^{n_{p 1} \times n_{w}}$ and $D_{y 1} \in \mathcal{R}^{n_{p y} \times n_{w}}$ are the corresponding disturbance distribution matrices, and $B_{2} \in \mathcal{R}^{n_{p} \times n_{p u}}$ and $D_{\infty 2} \in \mathcal{R}^{n_{p 1} \times n_{p u}}$ are the corresponding control distribution matrices, respectively.

The objective now is to find an optimal sensor combination where there exists a stabilizing dynamic outputfeedback controller $K(s)$ given by the following statespace expression

$$
\begin{aligned}
\dot{x}_{c}(t) & =A_{c} x(t)+B_{c} y(t) \\
u(t) & =C_{c} x(t)+D_{c} y(t)
\end{aligned}
$$

and where there exists a fault detection filter $F(s)$ given by

$$
\begin{aligned}
\dot{x_{f}}(t) & =A x_{f}(t)+B u(t)+L_{f}\left(y(t)-C x_{f}(t)\right), \\
z_{f}(t) & =H_{f}\left(y(t)-C x_{f}(t)\right),
\end{aligned}
$$

with filter gains $L_{f}$ and $H_{f}$ such that the following (RMS) performance index

$$
\rho:=\inf _{\substack{\lambda \infty<\gamma_{1} \\ \lambda \neq<\gamma_{0}}} \sqrt{\frac{\gamma_{1}^{2}}{2}+\frac{\gamma_{0}^{2}}{2}}
$$


with

$$
\lambda^{\infty}=\left\|T_{z_{\infty} w}\right\|_{\infty}, \text { and } \lambda_{-}^{\infty}=\frac{\left\|T_{z_{f} w}\right\|_{\infty}}{\left\|T_{z_{f} f}\right\|_{-}}
$$

is obtained among all candidate sensor combinations, where $T_{z_{\infty} w}$ is the transfer function from $w$ to $z_{\infty}$, $T_{z_{f} w}$ is the transfer function from $w$ to the residual $z_{f}$ and $T_{z_{f} f}$ is the transfer function from $f$ to the residual $z_{f}$, respectively. Although a number of different forms for $\rho$ can be chosen we adopt a root mean square approach, i.e. emphasizing the average of the magnitudes of $\gamma_{0}, \gamma_{1}$ quantities relating to control objective and fault detectability respectively.

Thus, the problem under consideration can be formulated as follows:

Problem 2.1: Let all variables be defined as above. Find an optimal sensor set $k \in \mathcal{S}$ with $\mathcal{S}$ representing the entire set of sensor combinations, such that $\rho_{k} \leq \rho_{i}, i=$ $1,2, \ldots, N$, where $\rho_{i}$ is the mixed norm performance index selected as in (10) with the corresponding sensor set $i$ deployed for a defined set of faults.

Remark 2.1: Note that the objective is in particular to find an appropriate sensor combination with the preferred control and fault detection properties, rather than directly to search for an optimal controller and an optimal fault detection filter for a given system. Our purposes is that the selected sensor configuration can be used as an effective basis prior to reconfiguration schemes ultimately leading to a complete fault tolerant system configuration. Undoubtedly the minimum possible set of sensors will be attractive in terms of reducing complexity in a practical system relative to maintenance as well as sensor equipment and installation costs.

Earlier work on similar concepts of (input)/output selection was mainly focused on evaluating a single performance index such as nominal performance, robust performance and robust stability applied to fault-free environment [9], [10]. In addition, work on the use of $\|\cdot\|_{\infty}$ and $\|.\|_{2}$ for placing sensor/(actuator) pairs is addressed in [11] but in an open loop sense applied to flexible structures. Observer-based fault detection filters have been extensively exploited during the past ten years, by which a residual signal is generated to provide fault signatures [12]-[15]. The observer effectively cancels the process dynamics and is sensitive only to disturbances and faults. The filter design objective is then to reduce the sensitivity to disturbances while maintaining a given level of sensitivity to faults. It is hence of interest to incorporate fault detectability into initial system design and output selection. However, it should be noted that the term "fault detectability" we are referring to in this paper is the robust performance of the effect of faults in the residual in terms of norms, which is rather different from the term defined by [16], [17].

\section{Controller AND FD Filter Synthesis Via LMIS}

An analytical solution of Problem 2.1 is not straightforward due to the difficulty of incorporating all available sensor sets into one controller design setup. Here, we follow a tractable suboptimal solution using an iterative procedure to evaluate the performance index(es) for each chosen sensor combination.

Multi-objective optimizations problems in the area of robust control have been well studied via generalized LMI treatment [18]. However, in our case the problem is simple to solve since designing the controller and fault detection filter is separate (which is possible in the case where nominal models are assumed [6]).

We first consider designing the controller, i.e. referring to the control performance index $\gamma_{1}$. With the plant $P$ and controller $K$ given in Section II, the closed-loop system has the realization in (12). By virtue of the Bounded Real Lemma [19], $A_{c l}$ is stable and $\left\|T_{z_{\infty} w}\right\|_{\infty}<\gamma_{1}$ if and only if there exists a symmetric $P$ with $P>0$ and

$$
\left[\begin{array}{ccc}
P A_{c l}+A_{c l}^{T} P & \star & \star \\
B_{c l}^{T} P & -\gamma_{1} I & \star \\
C_{c l \infty} & D_{c l \infty} & -\gamma_{1} I
\end{array}\right]<0
$$

where $\star$ denotes terms readily inferred from symmetry.

However, the matrix inequality in (13) cannot be solved directly by a convex optimization algorithm since nonlinear terms in the matrix inequalities will be encountered [18]. The following result gives a linearized formulation of the optimization problem of $\gamma_{1}$, of an analytical and tractable manner.

Lemma 3.1: [18] Let all variables be defined as above, then a stabilizing dynamic controller exists such that $\left\|T_{z_{\infty} w}\right\|_{\infty}<\gamma_{1}$ is achieved if there exists $X, Y, \hat{A}, \hat{B}$, $\hat{C}$ and $\hat{D}$ such that (14) is true.

Then, the stabilizing dynamic controller is given by

$$
\begin{aligned}
D_{c}= & \hat{D}, \\
C_{c}= & \left(\hat{C}-D_{c} C_{y} X\right) M^{-T}, \\
B_{c}= & N^{-1}\left(\hat{B}-Y B_{2} D_{c}\right), \\
A_{c}= & N^{-1}\left(\hat{A}-N B_{c} C_{y} X-Y B_{2} C_{c} M^{T}\right. \\
& \left.-Y\left(A+B_{2} D_{c} C\right) X\right) M^{-T},
\end{aligned}
$$

where square and nonsingular $M$ and $N$ should be chosen such that

$$
M N^{T}=I-X Y .
$$

For the design of FD filter which should achieve minimal $\gamma_{0}$, we adopt an optimal FD scheme which is maximally insensitive to disturbances for a given minimum level of sensitivity to faults. This so called mixed $\mathcal{H}_{-} / \mathcal{H}_{\infty}$ fault detection problem has been previously considered in [20]-[23], where partial solutions were given. Optimal solutions in frequency domain and in state space have been given in [7] and [24], respectively.

The next result gives an existence condition on finding minimal $\gamma_{0}$ such that $\frac{\left\|T_{z_{f} w}\right\|_{\infty}}{\left\|T_{z_{f}}\right\|_{-}}<\gamma_{0}$ in an LMI formulation.

Theorem 3.1: [24] Let all variables be as defined above. Assume that $n_{y} \geq n_{f},\left(A, C_{y}\right)$ is detectable and that $G_{f}(s): \stackrel{s}{=}\left[\begin{array}{c|c}A & B_{f} \\ \hline C_{y} & D_{f}\end{array}\right]$ has no extended imaginary 


$$
\begin{aligned}
T_{z_{\infty} w}:=T_{c l \infty} \stackrel{s}{=} & {\left[\begin{array}{c|c}
A_{c l} & B_{c l} \\
\hline C_{c l \infty} & D_{c l \infty}
\end{array}\right]=\left[\begin{array}{ccc}
A+B_{2} D_{c} C_{y} & B_{2} C_{c} & B_{1}+B_{2} D_{c} D_{y 1} \\
B_{c} C_{y} & A_{c} & B_{c} D_{y 1} \\
\hline C_{\infty}+D_{\infty 2} D_{c} C_{y} & D_{\infty 2} C_{c} & D_{\infty 1}+D_{\infty 2} D_{c} D_{y 1}
\end{array}\right] . } \\
& {\left[\begin{array}{cccc}
A X+X A^{T}+B_{2} \hat{C}+\left(B_{2} \hat{C}\right)^{T} & \star & \star & \star \\
\hat{A}+\left(A+B_{2} \hat{D} C_{y}\right)^{T} & A^{T} Y+Y A+\hat{B} C_{y}+\left(\hat{B} C_{y}\right)^{T} & \star & \star \\
\left(B_{1}+B_{2} \hat{D} D_{y 1}\right)^{T} & \left(Y B_{1}+\hat{B} D_{y 1}\right)^{T} & -\gamma_{1} I & \star \\
C_{\infty} X+D_{\infty 2} \hat{C} & C_{\infty}+D_{\infty 2} \hat{D} C_{y} & D_{\infty 1}+D_{\infty 2} \hat{D} D_{y 1} & -\gamma_{1} I
\end{array}\right]<0, } \\
& {\left[\begin{array}{cc}
X & I \\
I & Y
\end{array}\right]>0 . }
\end{aligned}
$$

axis zeros. Then there exists

$$
\left[\begin{array}{c}
D_{f}^{\dagger} \\
D_{f}^{\perp}
\end{array}\right] D_{f}=\left[\begin{array}{c}
I_{n_{f}} \\
0
\end{array}\right], \quad \operatorname{rank}\left(\left[\begin{array}{c}
D_{f}^{\dagger} \\
D_{f}^{\perp}
\end{array}\right]\right)=n_{y}
$$

and

$$
\gamma_{o}=\min \left\{\boldsymbol{\gamma}: \boldsymbol{Z} \in \mathcal{R}^{n \times\left(n_{y}-n_{f}\right)}, \quad \begin{array}{l}
\boldsymbol{S} \in \mathcal{R}^{n_{f} \times\left(n_{y}-n_{f}\right)}, \\
\boldsymbol{P}=\boldsymbol{P}^{T} \in \mathcal{R}^{n \times n},
\end{array}\right.
$$$$
\begin{gathered}
{\left[\begin{array}{ccc}
\boldsymbol{P}\left(A-B_{f} D_{f}^{\dagger} C_{y}\right)+\boldsymbol{Z}_{D_{f}^{\perp} C_{y}+\star} & \star & \star \\
\left(B_{1}-B_{f} D_{f}^{\dagger} D_{y 1}\right)^{T} \boldsymbol{P}+\left(D_{f}^{\perp} D_{y 1}\right)^{T} \boldsymbol{Z}^{T} & -\boldsymbol{\gamma}^{2} I & \star \\
D_{f}^{\dagger} C_{y}+\boldsymbol{S}_{D_{f}^{\perp} C_{y}} & D_{f}^{\dagger} D_{y 1}+\boldsymbol{S}_{D_{f}^{\perp} D_{y 1}} & -I
\end{array}\right]} \\
<0\}=: \gamma .
\end{gathered}
$$

Furthermore, for any $\gamma \geq \gamma_{o}$ there exists $\boldsymbol{P}, \boldsymbol{Z}$ and $\boldsymbol{S}$ such that the inequality in (17) is satisfied and such that the equation $\boldsymbol{P R}=\boldsymbol{Z}$ has a solution for $\boldsymbol{R}$.

The assumptions on the pair $\left(A, C_{y}\right)$ and the zeros of $G_{f}(s)$ are necessary for $\left\|T_{z_{f} f}\right\|_{-}>0$ and $A+L_{f} C_{y}$ stable.

By way of summarizing the results in [24] we give the following algorithm for the design of $\mathcal{H}_{-} / \mathcal{H}_{\infty}$ fault detection filter:

Algorithm 3.1:

- Define $D_{f}^{\dagger}$ and $D_{f}^{\perp}$ such that (16) is satisfied.

- Find $\gamma_{o}, \boldsymbol{P}, \boldsymbol{Z}$ and $\boldsymbol{S}$ that solve the LMI optimization in (17).

- Solve the equation $\boldsymbol{Z}=\boldsymbol{P R}$ for $\boldsymbol{R}$.

- Define $L_{f}$ and $H_{f}$ as in

$$
L_{f}=-B_{f} D_{f}^{\dagger}+\boldsymbol{R} D_{f}^{\perp}, \quad H_{f}=D_{f}^{\dagger}+\boldsymbol{S} D_{f}^{\perp} .
$$

Moreover, the following steps are important for sensor selection decision making:

Algorithm 3.2:

1) Define fault conditions for the problem setup.

2) Define sensor set.

3) Solve for stabilizing controller, i.e. find $\gamma_{1}$

4) Solve for FD filter as in Algorithm 3.1, i.e. find $\gamma_{0}$

5) Evaluate $\rho$ given by (10).

6) Update and goto step 2 if fault conditions unchanged.

7) Update and goto step 1 if fault conditions change.

The following section illustrates the approach via a numerical example.

\section{ILLUSTRATIVE NUMERICAL EXAMPLE}

We use a numerical example to illustrate the applicability of the proposed approach of sensor selection in dynamics systems. The system considered is a modified F16XL system [25], with the matrices of the linearized model given as follows

$$
A=\left[\begin{array}{cccc}
-0.0674 & 0.0430 & -0.8886 & -0.5587 \\
0.0205 & -1.4666 & 16.5800 & -0.0299 \\
0.1377 & -1.6788 & -0.6819 & 0 \\
0 & 0 & 1.0000 & 0
\end{array}\right]
$$

$$
B_{d e}=\left[\begin{array}{c}
-0.1672 \\
-1.5179 \\
-9.7842 \\
0
\end{array}\right], B_{w g}=\left[\begin{array}{c}
0.0430 \\
-1.4666 \\
-1.6788 \\
0
\end{array}\right]
$$


TABLE I

gains $L_{f}$ and $H_{f}$ as

$$
\begin{gathered}
L_{f}=\left[\begin{array}{cccc}
16.1 & 10.4 & -4.8 & 0.5 \\
404.4 & 169.5 & -131.9 & 6.1 \\
1485.6 & 778.1 & -455.8 & 21.6 \\
-0.3 & -0.4 & -0.7 & -0.7
\end{array}\right], \\
H_{f}=\left[\begin{array}{cccc}
-1.4945 & -0.1374 & 1 & 0 \\
0.1002 & -0.1598 & 0 & 1
\end{array}\right],
\end{gathered}
$$

and an optimal $\gamma_{0}=0.0666$.

The $\mathcal{H}_{\infty}$ controller is also given by (15) as

$$
\begin{gathered}
A_{c}=(1.0 e+005) *\left[\begin{array}{cccc}
-0.0164 & -0.0181 & 0.0707 & 0.0002 \\
-0.0144 & -0.0159 & 0.0622 & 0.0002 \\
0.1787 & 0.1972 & -0.7713 & -0.0024 \\
1.4345 & 1.6659 & -6.5447 & -0.1136
\end{array}\right], \\
B_{c}=(1.0 e+006) *\left[\begin{array}{cccc}
-0.0005 & -0.0000 & -0.0010 & -0.0045 \\
0.0190 & 0.0011 & 0.0027 & -0.0075 \\
0.0018 & 0.0006 & -0.0442 & -0.0117 \\
0.1325 & -2.3131 & -0.0272 & -0.0006
\end{array}\right], \\
C_{c}=\left[\begin{array}{ccccc}
178.3454 & 199.0380 & -779.1486 & -2.3767
\end{array}\right], \\
D_{c}=1.0 e-005 *\left[\begin{array}{llll}
-0.0029 & 0.0253 & 0.1460 & -0.0184
\end{array}\right],
\end{gathered}
$$

and an optimal $\gamma_{1}=1.0000$. Therefore, the performance index for output selection under full sensor set is $\rho=$ 0.7087 .

Then, we investigate the remaining sensor sets following the same performance index. Given that pitch rate and angle sensors are essential for the fault scenario assumed, i.e. fault detection, we retain the corresponding measurements $\left(y_{3}, y_{4}\right)$ in our output selection. The remaining part is to choose $y_{1}, y_{2}$ or neither. Note that if only fault $f_{3}$ or $f_{4}$ is assumed then it is essential to keep the pitch rate or pitch angle sensor respectively in the sensor set.

Now, assume that $y_{2}$ is removed from the full output set, we get the following fault detection filter as

$$
\begin{gathered}
L_{f}=\left[\begin{array}{ccc}
-123.3 & 0.3 & 0 \\
-469.0 & -37.0 & -0.9 \\
-5559.2 & -60.6 & -1.5 \\
23.9 & -1.7 & 0
\end{array}\right], \\
H_{f}=\left[\begin{array}{ccc}
-1.1999 & 1 & 0 \\
0.5093 & 0 & 1
\end{array}\right],
\end{gathered}
$$

with an optimal $\gamma_{0}=0.3048$ and the $\mathcal{H}_{\infty}$ controller as

$$
\begin{gathered}
A_{c}=(1.0 e+005) *\left[\begin{array}{cccc}
0.0030 & -0.1333 & -0.1159 & -0.0005 \\
0.0237 & -1.0638 & -0.9250 & -0.0012 \\
0.0908 & -4.0518 & -3.5229 & -0.0101 \\
0.1218 & -5.3736 & -4.6713 & -0.0358
\end{array}\right], \\
B_{c}=(1.0 e+005) *\left[\begin{array}{ccc}
0.0542 & 0.0094 & 0.0332 \\
-0.2537 & -0.0963 & 0.2255 \\
0.2068 & -1.1331 & -0.4612 \\
5.8596 & -1.7592 & 1.9613
\end{array}\right], \\
C_{c}=(1.0 e+004) *\left[\begin{array}{llll}
0.0801 & -3.5739 & -3.1074 & -0.0115
\end{array}\right], \\
D_{c}=1.0 e-005 *\left[\begin{array}{lll}
0.0031 & 0.1027 & 0.0036
\end{array}\right],
\end{gathered}
$$

with an optimal $\gamma_{1}=1.0017$. Therefore, the performance index for output selection under the sensor set $y_{1}, y_{3}, y_{4}$ is $\rho=0.7404$. Note also that the controller is designed relative to the sensor set used, i.e. both the controller and filter are assumed to using the same sensor information.

Hence, we continue in a similar way relative to other sensor selections and summarize the results in Table I.

Note from Table I that rows 1-4 relate to both faults $f_{3}, f_{4}$, while rows 5-6 and 7-8 only to fault $f_{3}$ and $f_{4}$ respectively. Thus, in a fault-free environment and for the
OUTPUT SELECTION PERFORMANCE INDEX CONSIDERING FAULTS

$$
f_{3}, f_{4}
$$

\begin{tabular}{|c||c|c|c|}
\hline Outputs & $\gamma_{1}$ & $\gamma_{0}$ & $\rho$ \\
\hline \hline$y_{1}, y_{2}, y_{3}, y_{4}$ & 1.0000 & 0.0666 & 0.7087 \\
\hline$y_{1}, y_{3}, y_{4}$ & 1.0017 & 0.3048 & 0.7404 \\
\hline$y_{2}, y_{3}, y_{4}$ & 1.0015 & 8.9798 & 6.3889 \\
\hline$y_{3}, y_{4}$ & 1.0000 & 10.3811 & 7.3746 \\
\hline$y_{1}, y_{3}$ & 1.0000 & 0.3016 & 0.7386 \\
\hline$y_{2}, y_{3}$ & 72.6503 & 0.0729 & 51.3716 \\
\hline$y_{1}, y_{4}$ & 1.0028 & 0.0729 & 0.7175 \\
\hline$y_{2}, y_{4}$ & 1.0006 & 8.9830 & 6.3912 \\
\hline
\end{tabular}

defined control problem formulation it is appropriate to choose a two-sensor set, apart from the set $y_{2}, y_{3}$ as it is still possible to have proper robustness properties to the disturbances affecting the aircraft (illustrated via the low values of $\gamma_{1}$ ). The situation changes once faults $f_{3}, f_{4}$ to monitor are considered. In such a case it is appropriate to use sensors $y_{1}, y_{3}, y_{4}$ rather than the full set as there is only a minimal effect on the value of $\rho$. Note that the aim is to choose the minimum set of sensors satisfying the objectives. This set can be used as a basis for further designs in a fault tolerant framework.

In addition, we consider a different set of potential sensor faults, i.e. $f_{1}$ and $f_{2}$ from the longitudinal and normal velocity sensors. By using the same procedure, the following Table II is drawn:

TABLE II

OUTPUT SELECTION PERFORMANCE INDEX CONSIDERING FAULTS

$$
f_{1}, f_{2}
$$

\begin{tabular}{|c||c|c|c|}
\hline Outputs & $\gamma_{1}$ & $\gamma_{0}$ & $\rho$ \\
\hline \hline$y_{1}, y_{2}, y_{3}, y_{4}$ & 1.0000 & 1.0021 & 1.0010 \\
\hline$y_{1}, y_{2}, y_{3}$ & 1.0008 & 73.2363 & 51.7907 \\
\hline$y_{1}, y_{2}, y_{4}$ & 1.0005 & 1.1712 & 1.0892 \\
\hline$y_{1}, y_{2}$ & 1.0005 & 75.3057 & 53.2538 \\
\hline$y_{1}, y_{3}$ & 1.0000 & 73.1785 & 51.7498 \\
\hline$y_{1}, y_{4}$ & 1.0028 & 0.0880 & 0.7118 \\
\hline$y_{2}, y_{3}$ & 72.6503 & 1.0164 & 51.3766 \\
\hline$y_{2}, y_{4}$ & 1.0006 & 1.1740 & 1.0908 \\
\hline
\end{tabular}

Similar to the previous case, in Table II rows 1-4 relate to both faults $f_{1}, f_{2}$, while rows 5-6 and 7-8 only to fault $f_{1}$ and $f_{2}$ respectively. Similar decisions as in the case of Table I can be followed here, again noting that the fault scenario is rather important in the decision making. For example, in the case where both faults $f_{1}, f_{4}$ to monitor are considered, we can select sensors $y_{1}, y_{2}, y_{4}$ rather than the full set as again there is a minimal effect on the value of $\rho$.

Moreover, it is possible to follow a combinatorial decision making procedure if necessary. Thus, select different optimal sets of sensors corresponding to different (appropriate) fault considerations and utilise these as bases in the design of a re-configurable (e.g. switching between the different controllers) scheme for fault tolerant systems. 


\section{CONCLUSION}

We discussed on a new setup to the output selection problem with consideration of fault detectability. The performance index for decision making investigated combines both an $\mathcal{H}_{\infty}$ controller design and an $\mathcal{H}_{-} / \mathcal{H}_{\infty}$ fault detection filter design. An iterative approach is then followed to testify different sensor sets under this performance index, with each of them solved by LMIs.

In this paper we emphasize the $\mathcal{H}_{\infty}$-norm that relates to control performance measure and $\mathcal{H}_{-} / \mathcal{H}_{\infty}$ that relates to fault detection performance measure. It is envisaged that the selected sensor configuration can be used as an effective basis prior to reconfiguration schemes ultimately leading to a complete fault tolerant system configuration. Undoubtedly the minimum possible set of sensors, in practical systems e.g. aerospace or railway applications, will be attractive in terms of reducing complexity relative to maintenance as well as sensor equipment and installation costs.

While the proposed output selection algorithm takes account of disturbances, and hence has some robustness properties against additive plant uncertainties, it does not explicitly consider the issue of robustness against uncertainty. This may be an important issue since the formulated schemes are model-based and operate in a closed-loop framework. Current work investigates integrated design of the controller and filter via matrix inequalities under uncertainty conditions.

\section{ACKNOWLEDGMENT}

This research was supported in part under the EPSRC project Grant Ref. EP/D063965/1 and BAE Systems, and also has been partially supported by the Ministry of Defence (MoD) through the Data \& Information Fusion Defence Technology Centre. The authors would also like to thank Dr Emmanuel Mazars for useful discussions.

\section{REFERENCES}

[1] M. V. D. Wal and B. D. Jager, "A review of methods for input/output selection," Automatica, vol. 37, no. 4, pp. 487-510, 2001.

[2] M. Basseville, A. Benveniste, G. Moustakides, and A. Rougee, "Optimal sensor location for detecting changes in dynamical behavior," IEEE Trans On Auto. Ctrl, vol. 32, December 1987.

[3] L. Traves-Massuyes, T. Escobet, and R. Milne, "Model-based diagnosability and sensor placement," in 12th International workshop on principles of diagnosis, 2001.

[4] M. Basseville and I. Nikiforov, Detection of Abrupt Changes: Theory and Application. Prentice-Hall, 1993.
[5] H. Niemann and J. Stoustrup, "Integration of control and fault detection: nominal and robust design," in IFAC Fault Detection, Supervision and Safety for Technical Processes, Hull, UK, 1997, pp. 341- 346.

[6] J. Stoustrup and H. Niemann, "Fault tolerant feedback control," in ECC, Porto, Portugal, 2001

[7] I. Jaimoukha, Z. Li, and V. Papakos, "A matrix factorization solution to the $\mathcal{H}_{-} / \mathcal{H}_{\infty}$ fault detection problem," Automatica, vol. 42, no. 11, pp. 1907-1913, 2006.

[8] J. Park, G. Rizzoni, and W. Ribbens, "On the representation of sensor faults in fault detection filters," Automatica, vol. 30, no. 11, pp. 1793-1795, 1994.

[9] M. V. D. Wal, P. Philips, and B. D. Jager, "Actuator and sensor selection for an active vehicle suspension aimed at robust performance," International Journal of Control, vol. 70, no. 5, pp. 703-720, 1998.

[10] M. V. D. Wal and B. D. Jager, "Selection of sensors and actuators for an active suspension control problem."

[11] W. Gawronski, "Simultaneous placement of actuators and sensors," Journal of Sound and Vibration, vol. 228, no. 4, pp. 915-22, 1999.

[12] X. Ding and P. Frank, "Fault detection via factorization approach," Systems \& Control Letts., vol. 14, pp. 431-436, 1990.

[13] P. M. Frank, "Fault diagnosis in dynamic systems using analytical and knowledge-based redundancy: A survey and some new results," Automatica, vol. 26, no. 3, pp. 459-474, 1990.

[14] P. M. Frank and X. Ding, "Survey of robust residual generation and evaluation methods in observer-based fault detection systems," $J$. Proc. Cont., vol. 7, no. 6, pp. 403-424, 1997.

[15] R. Patton, P. Frank, and R. C. (eds), Issues of Fault Diagnosis for Dynamic Systems. Springer, 2000.

[16] B. Liu and J. Si, "Fault isolation filter design for linear timeinvariant systems," IEEE Trans. Automatic Control, vol. 42, no. 5, pp. 704-707, 1997.

[17] M. Nyberg, "Criterions for detectability and strong detectability of faults in linear systems," Int. J. Control, vol. 75, no. 7, pp. 490-501, 2002.

[18] C. Scherer, P. Gahinet, and M. Chilali, "Multi-objective outputfeedback control via LMI optimization," IEEE Trans. Automatic Control, vol. 42, no. 7, pp. 896-911, 1997.

[19] S. Boyd, L. El Ghaoui, E. Feron, and V. Balakrishnan, Linear Matrix Inequalities in Systems and Control Theory. Philadelphia, PA: SIAM Publications, 1994.

[20] X. Ding, L. Guo, and P. Frank, "A frequency domain approach to fault detection of uncertain dynamic systems," in Proc. IEEE Conf. Dec. \& Control, San Antonio, TX, 1993, pp. 1722-1727, IEEE Press, New York.

[21] X. Ding, P. Frank, and L. Guo, "An approach to residual generator and evaluator design and synthesis," in Proc. of IFAC World Congress, Sydney, Australia, 1993.

[22] M. Hou and R. Patton, "An LMI approach to $\mathcal{H}_{-} / \mathcal{H}_{\infty}$ fault detection observers," in UKACC International Conference on Control. '96, England, 1996, pp. 305-310.

[23] F. Rambeaux, F. Hamelin, and D. Sauter, "Optimal thresholding for robust fault detection of uncertain systems," Int. J. Robust \& Nonlinear Control, vol. 10, pp. 1155-1173, 2000.

[24] Z. Li, I. M. Jaimoukha, and E. Mazars, "State space solution to the $\mathcal{H}_{-} / \mathcal{H}_{\infty}$ fault detection problem," in Proc. IEEE Conf. Dec. \& Control, San Diego, CA, USA, Dec 2006.

[25] R. Chen and J. Speyer, "Robust multiple-fault detection filter," Int. J. Robust \& Nonlinear Control, vol. 12, no. 8, pp. 675-696, 2002. 